\section{A trajetória e experiência dos Comitês de Prevenção da Mortalidade Materna do Paraná}

\section{Trajectory and experience of the Maternal Mortality Committees in Paraná}

Vânia Muniz Néquer Soares 1 Alaerte Leandro Martins 2

1,2 Secretaria Estadual de Saúde do Paraná. Rua Piquiri 170. Rebouças. Curitiba, PR, Brasil. CEP 80230-140 E-mail:vaniam@onda.com.br.

\section{Introdução}

A mortalidade materna, medida pela Razão de Mortalidade Materna (RMM) espelha a qualidade da assistência prestada à vida reprodutiva dos cidadãos e, indiretamente, o nível de desenvolvimento das regiões e países e a concepção de seus dirigentes, especialmente no que diz respeito à assistência à saúde da mulher. Essas afirmações podem ser comprovadas através dos diferentes índices encontrados para países desenvolvidos $(7,8 / 100.000$ nascidos vivos-NV) e em desenvolvimento, onde atingem níveis muito altos (230/100.000 NV na Bolívia, e 523/100.000 NV no Haiti) ${ }^{1}$, revelando que as Américas apresentam uma das maiores iniqüidades em mortalidade materna do mundo. ${ }^{2}$

A RMM para o Brasil em 2002, segundo dados do Ministério da Saúde, era de 53,4/100.000 NV. Utilizando-se o fator de correção/ajuste de taxas de 2,0 ou 1,4 , identificados respectivamente pelas pesquisas desenvolvidas por Tanaka ${ }^{3}$ e Laurenti et al.,4 a RMM corrigida estaria entre 106,8 e 74,76 mortes maternas por $100.000 \mathrm{NV}$, porém outras estimativas apontam taxas ainda maiores.

Trata-se de morte anunciada para as mulheres excluídas, na medida em que ocorre mais em países pobres e em desenvolvimento. Os estudos comprovam que acomete, especialmente, as mulheres de baixa renda e pouca escolaridade, principalmente na periferia dos centros urbanos. Para prevenir a morte materna, portanto, são necessárias medidas de melhoria da situação socioeconômica, especialmente renda e escolaridade, da formação dos profissionais que prestem assistência, garantia de parto seguro, organização do sistema de referência para atendi- mento as emergências obstétricas, dentre tantas outras. São imprescindíveis ações amplas e articuladas que visem uma mudança efetiva do atual modelo assistencial na área da obstetrícia, quem vem demonstrando deficiências inegáveis, evidenciada pelo elevado número de óbitos evitáveis por melhor assistência médica e hospitalar, cerca de $60 \%$ pelos dados obtidos no Paraná, por exemplo.

Como "a ponta de um iceberg", referido por Braga et al.,5 evidencia-se o número de casos das mulheres que chegaram a óbito por complicações da gravidez, parto e puerpério, e todo o volume do iceberg, submerso, é representado pela elevada morbidade materna e perinatal e as condições de vida, saúde e acesso a serviços de qualidade dessas mulheres, especialmente na fase reprodutiva. Tratase, então, de um indicador da eficácia de programas e da assistência obstétrica e da saúde da mulher, evidenciando de forma contundente as diferenças entre as regiões.

A falta da mulher e mãe no lar causa, em geral, a desestruturação da família, senão até de uma população que depende dela. ${ }^{1}$ Os lares se desfazem, as crianças ficam órfãs e acabam vivendo com tios ou avós, em instituições ou na rua. Trata-se de uma violência contra a mulher que pode gerar violência para os filhos.

Em maio de 1987, o V Encontro Internacional Mulher e Saúde, realizado na Costa Rica, mulheres de mais de 80 países, entre elas muitas brasileiras, lançaram uma Campanha Mundial pela Redução da Mortalidade Materna. Desde então a data foi consagrada como Dia Internacional de Ação pela Saúde da Mulher. Ainda em 1987, no Quênia, aconteceu a Conferência "Iniciativa para a Maternidade 
Segura", promovida pela Organização Mundial da Saúde (OMS), objetivando visibilizar a mortalidade materna e reduzi-la em $50 \%$ até o ano 2000.6

No Brasil, ao longo da década de 90, como resultado das lutas dos movimentos feministas e de profissionais de saúde comprometidos com os direitos humanos, o problema da mortalidade materna passou a fazer parte agenda política brasileira. Em 1993, o governo brasileiro tornou-se signatário das resoluções da $23^{\text {a }}$ Conferência Sanitária Pan-americana, acordando reduzir a mortalidade materna em $50 \%$ até o final do ano 2000.3,6

Neste contexto, com o apoio e incentivo do Ministério da Saúde, em 1988 realizou-se o "I Seminário Estadual sobre Mortalidade Materna no Estado do Paraná", por iniciativa de Luiz Fernando Cajado de Oliveira Braga, médico gineco-obstetra, professor da Universidade Federal do Paraná, que propôs, a partir de 1989, a implantação dos Comitês de Prevenção da Mortalidade Materna (CPMM) do Paraná: instituíram-se neste ano o Comitê Estadual e 22 comitês regionais, com o objetivo de identificar e estudar os óbitos maternos e apontar medidas de intervenção para sua redução, que permanecem até os dias atuais. ${ }^{7}$ É sobre a atuação desses Comitês que trata este Informe Técnico.

Comitês de Prevenção da Mortalidade Materna são organismos de natureza interinstitucional, multiprofissional e confidencial que visam identificar todos os óbitos maternos e apontar medidas de intervenção para a redução da mortalidade materna na região de abrangência. Essa iniciativa teve êxito em diferentes países no mundo, iniciando-se nos Estados Unidos na década de 30, e na Inglaterra na década de 50, expandindo-se para outros países. $\mathrm{Na}$ América Latina, a experiência mais antiga e exitosa é a de Cuba, iniciada em 1987. A partir de 1995, os comitês expandiram-se na América Latina, e cerca de 11 países criaram seus comitês nacionais. 8,9

A implantação de comitês é recomendada por organismos nacionais e internacionais, como uma das estratégias para a redução da mortalidade materna. 2,9

\section{A instalação e o funcionamento dos Comitês no Paraná}

Considerando-se que o principal objetivo dos Comitês de Morte Materna é mobilizar parceiros para a redução da mortalidade materna, além de identificar e analisar os óbitos maternos, classificálos em evitáveis ou inevitáveis e, principalmente, propor quais as medidas de prevenção, faz-se necessário dizer que os Comitês no Paraná foram instalados por definição política em 1989, com a implantação oficial em 1995, através da portaria 71/95 assinada pelo Secretário de Estado da Saúde e publicada em Diário Oficial, seguindo orientação da portaria 773, de 07/04/94, do Ministério da Saúde.7

Para garantia do pleno funcionamento, considerou-se fundamental, desde o princípio, o caráter interinstitucional, multiprofissional e confidencial, definidos em Regimento Interno, que também foi publicado em 1995 e atualizado em 2001. Dessa forma, compõem o Comitê Estadual em torno de 24 membros efetivos, assim distribuídos:

- Representantes da Secretaria Estadual de Saúde, setores de vigilância epidemiológica e assistência à saúde;

- Universidades (representantes das faculdades de medicina e enfermagem da Pontifícia Universidade Católica do Paraná (PUC, PR), da Universidade Federal do Paraná (UFPR), Faculdade Evangélica de Curitiba (FEPAR), Universidade Estadual de Londrina (UEL), Universidade Estadual de Maringá (UEM) e Universidade Estadual de Oeste do Paraná (UNIOESTE), dentre outras;

- Conselho Regional de Medicina e de Enfermagem;

- Associação Brasileira de Enfermagem - Seção Paraná, Associação Brasileira de Enfermagem Obstétrica - Seccional Paraná, Associação Médica do Paraná, Sociedade Paranaense de Ginecologia e Obstetrícia;

- Hospitais de referência de gestação de alto risco, Hospital de Clínicas da UFPR e Hospital Evangélico de Curitiba, Hospital Universitário de Londrina;

- Organizações Não Governamentais (ONGS) e Associações da sociedade civil: Associação Saza Lattes, Pastoral da Criança, Fórum Popular de Mulheres.

A mesma estrutura/composição é proposta para os Comitês das 22 Regionais de Saúde do Estado; o estado contava ainda, até 2003, com 151 Comitês municipais e 30 hospitalares. São definidas no Regimento as atribuições e o fluxograma de cada nível e o período de gestão, de dois anos, havendo no Comitê Estadual um revezamento da presidência entre médicos e enfermeiras, sendo livre para todos os membros se candidatarem a presidência:

- 1989-1994 - Luiz Fernando Cajado Oliveira Braga, médico, professor da UFPR

-1995-1997 - Vânia Muniz Nequer Soares, enfermeira da Secretaria Estadual de Saúde

-1998-1999 - Hélvio Bertolozzi Soares, médico, 
professor da UFPR

- 2000-2001 - Alaerte Leandro Martins, enfermeira, representante da ABEn - PR

- 2002-2003 - Fernando César Oliveira Júnior, médico professor da UFPR

- 2004- 2005 - Eliana Portella Carzino, enfermeira, professora da PUC-PR.

O CEPMM-PR funciona na sede da Secretaria Estadual de Saúde, vinculado ao Centro de Informação e Diagnóstico em Saúde (CIDS), que mantém sua infraestrutura, contando atualmente com uma enfermeira, dois ginecologistas e um estagiário, utilizando-se três computadores. As reuniões são mensais, com cronograma anual.

Ressalte-se que nos primeiros anos de implantação dos Comitês, o estado contou com financiamento do Ministério da Saúde, Organização Panamericana da Saúde e UNICEF, para a realização dos seminário anuais de avaliação e, em 1999, por ocasião dos dez anos dos Comitês, contou também como apoio da UNFPA (United Nations Population Fund).

O CEPMM-PR desenvolve atividades específicas de acordo com as funções/finalidades definidas pelo Ministério da Saúde para os Comitês:8-10 vigilância epidemiológica (investigação), educação/informação, divulgação/mobilização, normativa e assessoria, definidas em programação anual e bianual, relacionadas a seguir.

\section{Função de vigilância epidemiológica (investigação)}

Para garantir a identificação de todos os óbitos, foi institucionalizado o Sistema Estadual de Vigilância e Investigação do Óbito Materno, criando-se um sistema (em rede) próprio de vigilância epidemiológica nos 399 municípios do estado, independente da criação dos comitês, através de Resolução Estadual e posteriormente valendo-se também das Resoluções 256, de 01/10/97 e 1399/99, do Ministério da Saúde. 9

Utilizando-se a metodologia Reproductive Age Mortality Study (RAMOS), são investigados anualmente em torno de 3000 óbitos de mulheres em idade fértil (10 a 49 anos), cerca de $90 \%$ do total, identificando os óbitos maternos (média de 150/ano). A investigação é realizada pelas equipes dos serviços de vigilância epidemiológica municipais, através de pesquisa nos prontuários ambulatoriais e hospitalares, e entrevista com os profissionais que atenderam a cliente, e com familiares. Após a investigação, encaminham aos Comitês Regionais o
"Mapa de Óbitos de Mulheres em Idade Fértil', acompanhados das fichas de investigação confidencial de óbitos maternos confirmados com cópia do prontuário hospitalar e do pré-natal e das entrevistas com familiares e profissionais. Os comitês regionais analisam preliminarmente esses documentos e encaminham ao CEPMM, do Paraná.

No Comitê Estadual são processadas todas as informações, desde o controle do número esperado de óbitos de mulher em idade fértil de cada Regional de Saúde, o controle de todos os óbitos maternos investigados, sua digitação em programa informatizado próprio, e a elaboração dos estudos de casos individuais. Consta no Regimento que os Comitês Municipais e Regionais têm o prazo de dois meses para concluir a investigação dos óbitos maternos, o que nem sempre ocorre. O objetivo seria minimizar o tempo de conclusão do caso para retorno ao local de ocorrência, para que se identifiquem as estratégias que reduzam a possibilidade de novos casos.

Com o objetivo de identificar a totalidade dos óbitos maternos e para facilitar as investigações, foram criadas as "Fichas de Investigação de Óbito de Mulher em Idade Fértil" e a "Ficha de Investigação Confidencial de Morte Materna", atualmente na sua terceira versão. Em 1993 foram informatizadas todas as informações, criando-se banco de dados no programa Epi-Info; e posteriormente em programa Access.

Com os resultados das investigações e após análise de cada caso pelo comitê estadual referendado em Câmaras Técnicas pelos Comitês Regionais, desde 1990 é realizada a correção do Sistema de Informações em Mortalidade (SIM). Como pode ser observado na Figura 1, existe uma sub-notificação ainda presente da mortalidade materna (mortes maternas não declaradas), tendo-se para o período de 1990-1998 um Fator de Correção de 1,6, com uma sub-notificação em torno de 39\%, de 1,7 para o triênio $1997-1999,6$ e para o triênio 2000-2002, o fator de correção foi o mesmo, 1,7. Este fenômeno é, no entanto, universal, ocorrendo também nos países desenvolvidos, cujos sistemas de informação em saúde são de reconhecida credibilidade. A França apresentou para 1992-1994 uma RMM de 10/100.000 NV e fator de correção de 2,0 e o Canadá em 1993, RMM de 4/100.000 NV e fator de correção de 1,5.11

A frequiente sub-notificação de óbitos maternos é atribuída a razões diversas: desconhecimento das regras de preenchimento do atestado de óbito, desconhecimento de sua importância nas estatísticas vitais, ocultamento da causa real por possibilidade de associação a negligência ou imperícia no cuidado 


\section{Figura 1}

Razão de mortalidade materna ( por 1.000 .000 nascidos vivos).

Paraná, 1990 - 2003.

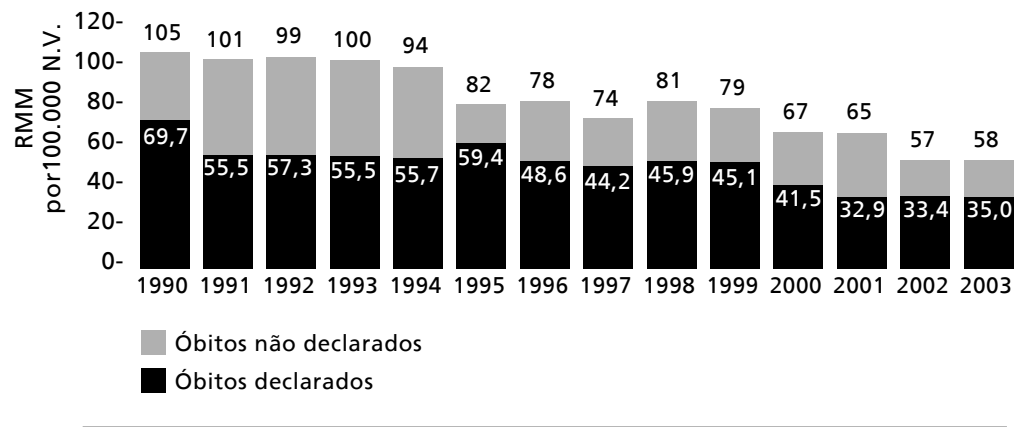

Fonte : Secretaria de Saúde, Paraná; 2003.

ou a ato ilegal.11

O estado conta com boa cobertura de notificação/registro de óbitos em geral, 95,6\% do estimado, portanto o sub-registro de óbitos de mulheres em idade fértil é praticamente nulo, mas permanece a sub-notificação da causa materna que só é identificada pelos Comitês.

Destaca-se como uma das ações relevantes desenvolvida pelo CEPMM-PR em meados de 19921993, para identificação de todos os óbitos maternos, o contato com a Secretaria de Estado de Segurança Pública, solicitando ampliação dos Serviços de Verificação de Óbitos (SVO) no estado e a solicitação de abertura e exploração da cavidade uterina nas necropsias de mulheres em idade fértil, para confirmação de estado gestacional, que até então não era rotina. Ao mesmo tempo, vários Comitês Regionais desenvolveram projeto para identificação de "cemitérios clandestinos", em parceria com as Seções de Vigilância Sanitária, garantindo, dentre outros, a identificação e o registro de todos os óbitos.

O Paraná apresentou uma redução gradativa da mortalidade materna até 2002, como demonstrado na Figura 1. A RMM de 57,96/100.000 NV, identificada em 2003 é considerada ainda relativamente alta se comparada às taxas dos países desenvolvidos, mas é um dado real, e uma das menores taxas do país, se aplicado o fator de ajuste nos outros estados.

\section{Função educativa/informativa}

Além da investigação dos óbitos maternos, uma das funções mais importantes dos Comitês no Paraná é a educativa/informativa, que desde o início foi priorizada inclusive com a divulgação de bibliografia pertinente, seja de saúde da mulher em geral e da mortalidade materna em particular, para os integrantes dos Comitês, aos profissionais interessados e universidades. Como exemplo, podemos citar a divulgação de textos, artigos e documentos oficiais, sobre direitos sexuais e reprodutivos, sobre a humanização do parto e nascimento, a divulgação dos protocolos de tratamento da Doença Hipertensiva Especifica da Gravidez, das Hemorragias pós-parto, dos estudos de casos, dos relatórios do Comitê Estadual, dentre outros.

Um Seminário Anual dos Comitês do Estado é realizado com o objetivo de capacitação das equipes, discussão das maiores dificuldades enfrentadas no cotidiano e para identificação conjunta das medidas de prevenção da mortalidade materna, introduzindose em cada evento temas importantes para discussão, dentre eles: a humanização da assistência ao parto, os direitos e a saúde sexual e reprodutiva, a redução de cesáreas, o pré-natal de qualidade, a assistência às emergências obstétricas. Com a criação também dos Comitês de Mortalidade Infantil, desde 2001 esse evento tem sido realizado em conjunto com os comitês regionais de mortalidade infantil.

Com o objetivo de facilitar a inclusão e discussão do tema "Mortalidade Materna" nos currículos dos cursos de medicina e de graduação em enfermagem, foi elaborado "Kit Aula sobre Mortalidade Materna", em 1995, distribuído para todas as universidades do estado. A campanha alusiva ao 28 de maio, realizada desde 1993, quando foi declarado como o "Dia Estadual de Prevenção da Mortalidade Materna", tem a definição de um tema gerador, que permanece por dois anos e é desenvolvido por meio de projeto envolvendo desde a confecção de folhetos, cartazes, jingles, teleconferências, até a realização de seminários a nível regional em alguns municípios. Os últimos temas foram:

- 1994 / 1995 - "Necessidade da assistência de pré-natal com mais qualidade"

- 1996 / 1997 - "Incentivo ao Parto Normal e necessidade da redução de casarias"

• 1998 / 1999 - "Parto Normal e Humanizado"

• 2000 / 2001 - "Prevenção da gravidez precoce e indesejada na adolescência", slogan: Gravidez na Adolescência Não é Brincadeira, com concurso e premiação de adolescentes escolares para definição de slogan, cartaz e música;

- 2002 / 2003 - "Atenção à Saúde Sexual e Reprodutiva na prevenção da Gestação de Alto Risco" 


\section{Estudos de caso - Câmara Técnica}

Mesclando a função epidemiológica com a função educativa, são realizadas anualmente duas câmaras técnicas para redução da mortalidade materna, quando são analisados os estudos de caso de óbitos maternos. A elaboração dos estudos de casos, aproximadamente 150 ao ano, teve início em 1994 e as câmaras técnicas foram implantadas em 1997, com a participação dos Comitês Regionais. A função epidemiológica se evidencia no que diz respeito à elaboração dos Cadernos/relatórios dos estudos de casos, a correção do sistema oficial de óbitos/SIM, e a função educativa na participação de vários profissionais dos Comitês e muitas vezes alunos da graduação e residentes de medicina das análises nas câmaras técnicas. É garantido o sigilo dos casos, tendo sempre um coordenador e relator de grupo que recolhem toda a documentação após cada caso concluído.

Além de garantir a uniformidade dos resultados, a realização das câmaras técnicas tem o objetivo de concluir os casos para possibilitar a retro-alimentação, devolução do caso concluído, com o mesmo caráter educativo, ao(s) profissional(is) que atendeu (ram) a cliente e ao diretor clínico do hospital, com análise e classificação consensual dos vários profissionais que integram os Comitês. Desde 2001, os estudos de casos recebem com assinatura de ciência formal do profissional e diretor clínico da instituição onde ocorreu o óbito para devolução ao comitê estadual.

Os estudos de caso são elaborados inicialmente por dois médicos gineco-obstetras da equipe técnica do comitê estadual, a partir dos documentos (prontuários, fichas de investigação, entrevista) enviados pelos comitês municipais e regionais. $\mathrm{O}$ estudo de caso consiste na descrição de toda história clínica e na análise e classificação do óbito materno, que se constitui na:

1. Definição da causa básica do óbito;

2. Classificação da morte em obstétrica direta, indireta, não obstétrica e tardia;

3. Definição se o óbito materno está declarado ou não na declaração de óbito;

4. Definição da evitabilidade do óbito;

5. Definição das responsabilidades pelo óbito e;

6. Definição das medidas de prevenção.

\section{Conceituação adotada na câmara técnica}

Morte Materna Declarada: quando a causa especificada na Declaração de Óbito (DO) menciona clara- mente e por extenso o estado gravídico-puerperal por ocasião do óbito, por incluir um dos diagnósticos relacionados no Capítulo XV da Classificação Internacional de Doenças (CID) ou por fazer qualquer menção ao estado gestacional na parte 2 da DO, independente de estarem ou não preenchidos os campos 43 e 44 . Esse conceito foi mudado a partir de 2004, considerando o preenchimento desses campos também como morte materna declarada ${ }^{9}$.

-Evitabilidade: conforme recomendação do Ministério da Saúde, ${ }^{7}$ deve-se aplicar um critério de evitabilidade baseado não só no diagnóstico e na análise da pertinência das medidas terapêuticas adotadas, mas também na disponibilidade dos serviços de atenção materna e obstétrica especializada e a distância do local de residência habitual da grávida, nas facilidades existentes de transporte das mesmas ao local de atenção, nas condições de eficiência e capacidade resolutiva dos serviços disponíveis e outros antecedentes clínicos e sociais da falecida.

-Responsabilidade:

Médica: quando ocorre erro por não obediência às normas e protocolos assistenciais, falta de conhecimento científico do profissional que presta assistência, negligência, incompetência do profissional de qualquer formação, seja médico, enfermeiro ou outro e/ou aplicação incorreta de medicamentos por pessoas não qualificadas.

Hospitalar: são os também chamados erros administrativos, quando as condições ambulatoriais ou hospitalares são insuficientes e inadequadas. Incluem-se nesse item acesso e infraestrutura.

Da paciente: quando ocorrer da paciente, mesmo sendo informada, não comparecer ao Serviço de Saúde, estando esse acessível, ou quando ela não se sujeita aos exames solicitados, ou ainda, não cumpre a terapêutica prescrita.

Social: é a responsabilidade coletiva de toda uma comunidade ou nação, envolvendo as condições sociais, econômicas, políticas. Incluem-se os casos decorrentes da miséria, do analfabetismo, da falta de informação, desnutrição, endemias, etc.

-Medidas de prevenção

Melhor Assistência Médica: relacionada às mortes evitáveis por maiores estudos, ponderação e cuidados dos profissionais que prestaram assistência, o que evitaria repetição de circunstâncias seme-lhantes. Esses erros deveriam merecer maior atenção dos diretores de maternidades e dos órgãos de classe. Recomenda-se 
também estímulo à atualização do conhecimento e troca de opiniões e experiências e organização de grupos de estudo da mortalidade nos serviços para discutir todos os casos de óbito.

-Melhor Assistência Hospitalar: as condições dos Serviços de Saúde, seus equipamentos e funcionamento estão interligados com a equipe técnica de profissionais e a do hospital que administra, coordena, supervisiona, providencia recursos e pessoal, supre deficiências e aponta soluções.

-Medidas Educativas e Informativas: no caso de responsabilidade da paciente, para evitar que a mesma se recuse em participar do pré-natal e tratamentos indicados, são necessárias medidas de educação e saúde e envolvimento de familiares para percepção da importância do acompanhamento médico durante a gestação.

- Medidas Sociais: medidas sociais como a melhoria de acesso aos serviços, investimento em educação das adolescentes, e melhores condições de vida, podem reduzir um certo número de mortes maternas que ocorrem nas camadas mais empobrecidas da população.

Os critérios de responsabilidade e medidas de prevenção foram adaptados da classificação da Associação Médica Americana. A classificação da causa básica do óbito e principalmente a evitabilidade e responsabilidade (correspondente às falhas do atendimento) muitas vezes têm aspectos subjetivos. Para aumentar a sua validade é que os Comitês devem ser multiprofissionais, além de contar com o parecer de vários médicos; a multiplicação de opiniões diminui a subjetividade e reforça as decisões.

Passam pela câmara técnica todos os casos que envolvam mulheres em estado gestacional, inclusive os casos de AIDS, câncer, entre outros, para definição se maternos ou não, assim como os tardios. Acompanha-se também uma série de casos de morte materna não obstétrica preocupantes, como casos de suicídio, ou homicídios cometidos pelo companheiro, quando relacionados ao diagnóstico da gravidez; e os casos de acidentes de trânsito ou outros. Enfim, todos os óbitos de mulheres em idade fértil devem ser investigados e, se confirmado que a mulher quando faleceu estava grávida, era parturiente ou puérpera, devem ser analisados em câmara técnica.

No entanto, tem-se utilizado para o cálculo da RMM apenas os óbitos diretos e indiretos, até 42 dias pós-parto, conforme preconiza a OMS, os óbitos tardios e acidentais ou incidentais são computados separadamente.

\section{Função de divulgação}

Foram sistematicamente disponibilizados relatórios anuais sobre a Situação da Mortalidade Materna no Paraná de 1990 a 1993, e a partir de 1994 passaram a ser elaborados e disponibilizados, relatórios trienais: triênio 1994-96, 1997-99, 20002002.5,7,12,13 Entendeu-se que o perfil da mortalidade materna não se altera significativamente ano a ano e a análise trienal, dado o maior volume de dados, permite conclusões mais concretas para a tomada de novas diretrizes, seguindo a tendência mundial, embora os dados anuais sejam divulgados pela internet, nos seminários anuais de avaliação e estejam disponíveis no comitê para consulta. Ainda na função de divulgação, pode-se incluir:

- A disponibilização dos estudos de caso (sem identificação), para a comunidade científica;

- A disponibilização dos estudos de caso (identificados), para profissionais que atenderam a cada caso;

- A elaboração de artigos em periódicos, materiais para mídia e entrevistas;

- A criação do boletim do Comitê Estadual "Vigiar para Proteger";

- A criação da página da Internet do Comitê no site da Secretaria Estadual de Saúde

- Os estudos específicos: Situação das Cesáreas no Estado, Doença Hipertensiva Específica na Gravidez, Hemorragias, entre outros, apresentados em eventos científicos e no estado;

- Os trabalhos científicos elaborados pelos comitês regionais e municipais.

\section{Função normativa}

O Comitê Estadual, tendo uma composição de profissionais diversificados e com respaldo de diversas entidades e sociedades científicas que representam e baseando-se no perfil epidemiológico identificado através das investigações, elaborou protocolos, pareceres, resoluções de relevância para melhoria da assistência, entre eles:

- Protocolos para acompanhamento de gestantes hipertensas e manejo da eclâmpsia;

- Protocolo sobre conduta em casos de hemorragias obstétricas;

- Parecer sobre incidência de cesáreas no Estado;

- Resolução sobre a presença do acompanhante e parto humanizado, aprovada pelo Conselho Estadual de Saúde;

- Resolução sobre a obrigatoriedade da utilização do partograma, vinculada à emissão de 
Autorização de Internação Hospitalar (AIH) para parto, também aprovada pelo Conselho Estadual de Saúde.

\section{Função de assessoria}

Compete ao Comitê Estadual assessorar aos gestores de saúde sempre que solicitado e aos comitês regionais permanentemente, podendo-se citar como ações neste aspecto:

- A participação na elaboração do Plano Estadual de Redução de Mortalidade Materna (Protegendo a Vida e Rede de Proteção a Vida) e municipais (Projeto Mãe Curitibana);

- A elaboração e distribuição do Kit Eclâmpsia para as maternidades (2002);

- Assessoria sistemática aos Comitês Regionais e quando solicitado a outros Comitês estaduais.

Dentre as atividades de assessoria, destaca-se o desenvolvimento do Projeto de Fortalecimento dos Comitês Regionais de Prevenção da Mortalidade Materna. No planejamento para o ano 2000, incluiuse a realização de reuniões/seminários nas Regionais de Saúde, com o objetivo de fortalecer e estimular os Comitês Regionais e os Municipais, atendendo a uma das propostas da "Carta do Paraná", elaborada no Seminário dos Dez Anos dos Comitês de Mortalidade Materna, realizado em agosto de 1999.7

Avaliando o resultado da mortalidade materna de 2002 e pelos resultados obtidos com o projeto em 2000, definiu-se por retomar o Projeto de Fortalecimento dos Comitês Regionais, para 2003 e 2004. A metodologia utilizada consiste em reuniões descentralizadas em cada Comitê Regional, com a participação de dois representantes do CEPMM-PR, e a presença sempre de pelo menos um ginecologista, objetivando:

- Discutir a redução da mortalidade materna em nível regional;

- Fazer o levantamento de dificuldades e avaliar a situa-ção de cada comitê;

- Elaborar conjuntamente Planos Regionais de Ação para Redução da Mortalidade Materna com metas pactuadas.

Para facilitar o processo, é encaminhado às Regionais de Saúde um modelo de projeto, solicitando o nome do contato que organizaria a reunião, a proposta de data e principalmente proposta de tema técnico de interesse da Regional de Saúde, sendo indicados alguns pré-selecionados pelo CEPMMPR, conforme a realidade local, como: partograma, redução de cesáreas, referência à gestação de risco. Dependendo da organização de cada Regional, a participação nas reuniões tem sido entre 20 a 300 pessoas.

\section{Considerações finais}

Foram conquistas dos Comitês no Paraná neste quinze anos (1989 a 2003), a correção sistemática e permanente da sub-notificação dos óbitos maternos, a gradativa redução da mortalidade materna no estado, a permanente parceria dos membros/instituições que constituem os comitês articulada num espaço participativo e democrático, dentre outras. A premiação oferecida pelo Ministério da Saúde em 2001, durante a I Mostra Nacional de Experiências Bem Sucedidas em Epidemiologia foi para o Comitê um forma de reconhecimento da efetividade do trabalho realizado pelos membros dos comitês estadual, regionais e municipais do estado.

Na perspectiva de assessorar os gestores estadual e municipais nas questões relacionadas à avaliação da assistência à saúde materna oferecida pelos prestadores de serviços do SUS, foi criada por solicitação do Comitê Estadual e aprovada pelo Secretário Estadual de Saúde, a "Comissão Estadual de Controle de Qualidade de Assistência à Saúde Materna" (CECQA), através das Resoluções 336 e 0113/2003. A ação dessa Comissão de Qualidade se dá dentro dos serviços, e inicia-se por demanda dos comitês, dos gestores, da ouvidoria ou auditoria e ainda "ex-ofício" ou por denúncia, determinando uma investigação in loco nos serviços com maior mortalidade materna, com vistas a correção de deficiências e melhoria da assistência. Esta é mais uma proposta do CEPMMM que deverá ser implementada.

Apesar de algumas dificuldades nesta trajetória, os Comitês no Paraná nunca tiveram períodos de interrupção nestes quinze anos, ao contrário de muitos comitês de outros estados brasileiros, demonstrando seu reconhecimento força e articulação. 10,14

Essas e outras conquistas demonstram a inserção ético-política dos Comitês no Paraná no período referido, respeitando o seu projeto inicial de buscar garantir uma maternidade segura às mulheres paranaenses. Novos avanços dependerão da correção de falhas identificadas em todo o processo e definição de novas estratégias que aperfeiçoem os trabalhos dos Comitês no Paraná. 


\section{Referências}

1. OPS (Organización Panmericana de la Salud), OMS (Organización Mundial de la Salud). Género, salud y desarrollo en las Américas .Ind básicos 2005.

2. OPS (Organização Panamericana de la Salud), OMS (Organización Mundial de la Salud). Resolucíon CSP26/14: Estratégia Regional para la Reducción de la Mortalidad y Morbilidad Maternas. Washington (DC); 2002.

3. Tanaka ACA. Dossiê mortalidade materna. Rede Nacional Feminista de Saúde e Direitos Reprodutivos, 2001. Disponível em http: www.redesaude.org.br [2006 jun 29]

4. Laurenti R, Mello JMHP, Gotlieb SLD. Mortalidade materna nas capitais brasileiras: algumas características e estimativa de um fator de ajuste. Rev Bras Epidemiol 2004; 7: 449-60.

5. Braga LFCO, Soares VMN, Ribeiro C, Nazareno ER. Relatório do Comitê de Mortalidade Materna do Paraná, 1991. Inf Epidemiol SUS. 1992; 7: 31-49.

6. Araújo MJO. Campanha Internacional Contra a Mortalidade Materna completa 13 anos. J Rede Fem Saúde 2000; 2 : 3-4.

7. CEPMM-PR (Comitê Estadual de mortalidade Materna do Paraná). Histórico dos Comitês no Paraná: dez anos de atuação. In: Anais do Seminário dos X Anos dos Comitês de Prevenção da Mortalidade Materna do Paraná; 1999 1214 ago.; Curitiba, Paraná. Curitiba: Secretaria Estadual de Saúde; 2001

Recebido em 11 de novembro de 2005

Versão final apresentada em 13 de outubro de 2006

Aprovado em 20 de outubro de 2006
8. Ministério da Saúde. Secretaria de Assistência à Saúde. Departamento de Assistência e Promoção à Saúde. Coordenação Materno-Infantil. Manual dos Comitês de Mortalidade Materna. Brasília (DF); 1994.

9. Ministério da Saúde. Secretaria Técnica de Políticas de Saúde. Área Técnica de Saúde da Mulher. Manual dos Comitês de Mortalidade Materna. 2. ed. Brasília (DF); 2001.

10. Siqueira AAF, Rodrigues AV. Uma análise da implantação dos Comitês de Estudos de Morte Materna no Brasil: um estudo de caso do Comitê do Estado de São Paulo. Cad Saúde Pública 2003; 19: 183-9.

11. Volochko A. A mensuração da mortalidade materna no Brasil. In: Berquó E, organizadora. Sexo e vida: panorama da saúde reprodutiva. Campinas: Ed. UNICAMP; 2003.

12. Soares HB, Soares VMN, Carzino E, Araújo RA. Mortalidade materna no Paraná, do anonimato à ação: relatório trienal 1994-96. Rev Ginecol Obstet 1998; 9: 7081.

13. CEPMM-PR. (Comitês de Prevenção da Mortalidade Materna do Paraná). Mortalidade materna no Paraná: vigilância e redução: relatório trienal 1997-1999. Curitiba: Secretaria Estadual de Saúde; 2002.

14. TCU (Tribunal de Contas da União). Monitoramento e prevenção da mortalidade materna: relatório de auditoria de natureza operacional. Brasília (DF); 2001. 